\title{
Rol del comunicador organizacional en Uruguay: tareas y funciones
}

\section{The role of the organizational communicator in Uruguay: tasks and functions}

\author{
Mónica Arzuaga-Williams
}

Cómo citar este artículo:

Arzuaga-Williams, Mónica (2020). "Rol del comunicador organizacional en Uruguay: tareas y funciones". Profesional de la información, v. 29, n. 3, e290326.

https://doi.org/10.3145/epi.2020.may.26

Artículo recibido el 10-04-2019 Aceptación definitiva: 18-12-2019

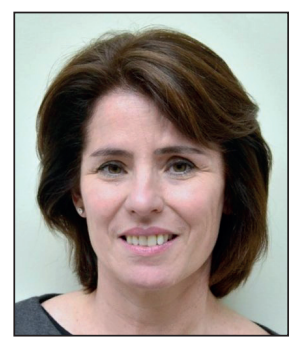

Mónica Arzuaga-Williams

https://orcid.org/0000-0002-1193-287x

Universidad de Málaga, Málaga, España

monicaarzuaga@uma.es

Universidad Católica del Uruguay

Montevideo, Uruguay

marzuaga@ucu.edu.uy

\section{Resumen}

Se presentan los resultados de una encuesta a 215 profesionales de la Comunicación Organizacional en Uruguay. Se describen de manera comparada los roles y tareas de los profesionales que trabajan en diferentes tipos de organizaciones, y de quienes se desempeñan como consultores o proveedores de servicios en comunicación organizacional. En particular se estudian los factores que inciden en la evaluación de la prestación de los servicios desde la perspectiva de clientes y de consultores. Se estudian las funciones de la Comunicación Organizacional y la percepción sobre sus resultados. Se concluye que la carrera profesional, el tipo de organización y el sexo del profesional inciden en el tipo de rol y las tareas que se desempeñan. Se observan diferencias en la percepción que clientes y consultores tienen sobre su relación. El predominio de las funciones de Comunicación Organizacional externa debilita los resultados alcanzados en comunicación interna.

\section{Palabras clave}

Comunicación Organizacional; Comunicación Corporativa; Relaciones Públicas; Comunicación interna; Roles profesionales; Profesionales; Consultores; Género; Función de la comunicación; Uruguay.

\begin{abstract}
This article presents the results of a survey of 215 organizational communication professionals in Uruguay. It describes in a comparative way the roles and tasks of professionals working in different types of organization versus those who work as consultants or service providers in organizational communication. In particular, the factors that affect the evaluation of the provision of services from the perspective of clients and consultants are studied. In addition, the functions of organizational communication and the perception of their results are examined. It is concluded that the professional career, the type of organization, and the sex of the professional affect the type of role and professional tasks. There are differences in the perception that clients and consultants have about their relationship. The predominance of external organizational communication functions weakens the results achieved in internal communication.
\end{abstract}

\section{Keywords}

Organizational Communication; Corporate Communication; Public Relations; Internal communication; Practitioner roles; Professionals; Consultant roles; Genre; Communication function; Uruguay. 


\section{Introducción}

En ocasiones se debate sobre los términos "Comunicación Organizacional” y "Relaciones Públicas" para denominar este campo profesional y sus diferencias. Aunque en algunos países predomina el uso del término "Relaciones Públicas", es "Comunicación Organizacional" el que en América Latina se propone con más frecuencia para expresar el campo disciplinar y el que incluye todas las expresiones, modalidades y aplicaciones de la comunicación para todo tipo de organizaciones (Krohling-Kunsch, 2011, p. 73).

De manera similar, Van-Ruler (2004) señala que, aunque en Estados Unidos y en algunos países europeos prevalece el uso del término "Relaciones Públicas", el concepto de "Gestión de la comunicación" es el que define de manera más adecuada el campo, por lo que pueden ser usados como equivalentes.

En este artículo se utiliza el término "Comunicación Organizacional” como genérico, sin discutir su demarcación epistemológica con el término "Relaciones Públicas" y otros de uso menos frecuente como "Comunicación Corporativa" o "Institucional”.

En una revisión de bibliografía científica realizada en la colección principal de Web of Science se observa que la mayoría de los artículos que se ocupan del rol del profesional de la Comunicación Organizacional presentan investigaciones realizadas en Estados Unidos, Reino Unido y, en menor medida, en otros países europeos, mientras que sólo un pequeño número de artículos presentan ensayos teóricos o investigaciones sobre el tema en América Latina (Arzuaga-Williams, 2019, pp. 141-142). En este sentido, Mellado y Barría afirman que

"la abundante investigación sobre los roles profesionales en Relaciones Públicas desarrollada en Estados Unidos y Europa contrasta con los escasos trabajos teóricos y empíricos que existen sobre estos temas en América Latina" (Mellado; Barría, 2012, p. 446).

A diferencia de las investigaciones realizadas en Estados Unidos y Europa, que se ocupan de muy variados temas sobre el desempeño del rol de los profesionales (Arzuaga-Williams, 2017), en América Latina las pocas investigaciones con evidencia empírica se centran casi exclusivamente en el aporte del profesional para los fines sociales u organizacionales.

Por un lado, las investigaciones que priorizan la función político-social de los profesionales definen sus roles según tengan aporte al bienestar de los empleados, la ética y responsabilidad social, el bienestar de la comunidad o la armonía con el gobierno. En esta perspectiva del conocido como modelo colaborativo se sitúan las investigaciones de:

- Molleda y Suárez (2003) en Colombia a 166 profesionales;

- Molleda y Ferguson (2004) en Brasil a 148 profesionales (Arzuaga-Williams, 2019, pp. 144- 145);

- el análisis comparativo realizado con resultados de la Macroencuesta Latinoamericana de Comunicación y Relaciones Públicas por Molleda et al. (2010) en los que se distingue además si los cuatro roles sociales de los profesionales aportan a los objetivos internos o externos de la organización.

Tabla 1. Principales roles según aporte social y a los objetivos organizacionales

\begin{tabular}{|l|c|c|c|c|}
\cline { 2 - 4 } \multicolumn{1}{c|}{} & $\begin{array}{c}\text { Aporta al bienestar de } \\
\text { los empleados }\end{array}$ & $\begin{array}{c}\text { Aporta a la ética y la } \\
\text { responsabilidad social }\end{array}$ & $\begin{array}{c}\text { Aporta al bienestar de } \\
\text { la comunidad }\end{array}$ & $\begin{array}{c}\text { Aporta a la armonía con } \\
\text { el gobierno }\end{array}$ \\
\hline $\begin{array}{l}\text { Aporta a los objetivos inter- } \\
\text { nos de la organización }\end{array}$ & $\mathrm{X}$ & $\mathrm{X}$ & \\
\hline $\begin{array}{l}\text { Aporta a los objetivos ex- } \\
\text { ternos de la organización }\end{array}$ & & & $\mathrm{X}$ & $\mathrm{X}$ \\
\hline
\end{tabular}

Por otro lado, están las investigaciones centradas exclusivamente en analizar el aporte estratégico de los profesionales a las organizaciones. Entre ellas destacan las de:

- Salas-Forero (2013) en 23 empresas privadas grandes de Colombia;

- Preciado-Hoyos, Nivia-Flórez y Correales-Rivas (2017) con 55 directivos de agencias de Comunicación Organizacional de Colombia;

- Krohling-Kunsch (2004) con 71 profesionales de organizaciones de diferente tipo en Brasil.

En ninguna de ellas se propone una tipología de roles profesionales. También el Latin American Communication Monitor ha generado datos en forma sistemática para varios países, sobre la función estratégica del rol profesional. http://latincommunicationmonitor.com/site/informes

Por último, Mellado y Barría (2012), también enfocados en estudiar el aporte estratégico del profesional, realizan una investigación en Chile en la que clasifican los roles profesionales en cuatro tipos según el estilo de gestión del profesional y el tiempo que dedica a la planificación (rol estratégico a largo plazo y rol técnico a corto plazo), así como el tipo de mediación del profesional y su actitud como asesor (rol activo-vigilante y rol pasivo-complaciente).

Como se observa, hasta la fecha no se ha publicado en América Latina una investigación con evidencia empírica sobre todos los roles de los profesionales de la Comunicación organizacional que incluya todas sus tareas y funciones, que aplique a todo tipo de organización y en todos los niveles jerárquicos. 


\section{Roles del profesional}

Broom y Dozier, autores de las primeras investigaciones empíricas en Estados Unidos sobre los roles de los profesionales y creadores del modelo más influyente, señalan que el desempeño profesional en este campo se puede sintetizar en dos roles principales (Broom; Dozier, 1986):

- gerente de comunicación: que realiza las tareas de asesorar y prescribir sobre los asuntos clave para la organización, facilitar los procesos de comunicación y facilitar la resolución de problemas;

- técnico en comunicación que se ocupa principalmente de producir contenidos o materiales, así como de la relación con los medios.

A partir de investigaciones en Reino Unido y Estados Unidos, Moss, Newman y DeSanto completan el modelo con los contenidos de las tareas que desempeñan los profesionales de la comunicación que ocupan niveles directivos. Los autores encuentran que, además de las tareas planteadas por Broom y Dozier, los gerentes de comunicación también desempeñan tareas propias del técnico de comunicación, como diseñar y producir materiales o estar en contacto con los medios (Moss; Newman; DeSanto, 2005, pp. 879-881).

Una situación particular se refiere a la escasa atención que los investigadores le han dado al componente creativo del rol profesional. Por lo general se incluye dentro de los roles técnicos y, según estudios exploratorios, este componente produce alto nivel de satisfacción en algunos profesionales (Dozier; Broom, 2012, p. 164).

Van-Ruler et al., por su parte, proponen cuatro roles principales y así definen qué implica cada uno (Van-Ruler et al., 2004, pp. 53-54):

- reflexivo: analiza, discute sobre valores y fines de manera que permita definir la misión y las estrategias organizacionales;

- gerencial: desarrolla planes, ejecuta las estrategias organizacionales, mantiene relaciones internas y externas de manera tal que generen confianza y entendimiento mutuo;

- operacional: prepara medios de comunicación, produce contenidos para los medios que permiten ejecutar servicios y planes llevados a cabo por otros;

- educacional: apoya a los miembros de la organización en el desarrollo de sus competencias comunicacionales para facilitar la comunicación entre ellos, y aporta a la evolución de las relaciones y la comunicación interna (tabla 2).

Tabla 2. Principales modelos de roles del profesional con sus tareas

\begin{tabular}{|c|c|c|}
\hline Broom; Dozier (1986) & Moss et al. (2000) & Van-Ruler et al. (2001) \\
\hline $\begin{array}{l}\text { Gerente (o director) de comunicación: } \\
\text { - Prescribe de forma experta } \\
\text { - Facilita procesos de comunicación } \\
\text { - Facilita procesos de resolución de pro- } \\
\text { blemas } \\
\text { Técnico en comunicación }\end{array}$ & $\begin{array}{l}\text { Gerente (o director) de comunicación: } \\
\text { - } \text { Asesora en políticas clave y estratégicas } \\
\text { - Monitorea y evalúa } \\
\text { - } \text { Gestiona con experticia asuntos clave } \\
\text { - } \text { Media entre intereses } \\
\text { - } \quad \text { Soluciona problemas } \\
\text { - } \quad \text { Diseña, produce materiales } \\
\text { - Contacta con los medios }\end{array}$ & $\begin{array}{l}\text { Reflexivo } \\
\text { Analiza, discute para definir la misión y las } \\
\text { estrategias } \\
\text { Gerencial } \\
\text { Desarrolla planes, ejecuta estrategias, mantie- } \\
\text { ne relaciones } \\
\text { Operacional } \\
\text { Prepara medios, produce contenidos } \\
\text { Educacional } \\
\text { Apoya, desarrolla competencias en otros }\end{array}$ \\
\hline
\end{tabular}

\subsection{Características de los roles}

Los roles profesionales en Comunicación Organizacional no son mutuamente excluyentes, es decir, una persona puede desempeñar principalmente un rol, pero también realizar tareas de otro (Dozier; Broom, 2012, pp. 141-142). Esto ocurre principalmente entre los profesionales que se encuentran en niveles jerárquicos altos (DeSanto; Moss, 2005, pp. 188191; Yeo; Sriramesh, 2009, pp. 423-424).

Si se analiza la vida de un profesional, se observa que los roles son dinámicos. Una misma persona puede cambiar el rol que desempeña según los años de experiencia y cómo evoluciona su carrera profesional (Dozier; Broom, 2012; Vieira; Grantham, 2015). También el desempeño del rol varía de acuerdo con la socialización que le imprime cada lugar de trabajo durante la carrera del profesional (Berkovitz; Hristodoulakis, 1999, p. 100).

Por otra parte, los roles son contingentes, es decir, ocurren unos u otros según diferentes factores organizacionales, sociales y personales. Un profesional puede desempeñar un rol distinto según su ubicación jerárquica dentro de la organización, si se encuentra más o menos cerca del lugar donde se toman las decisiones clave de la organización, o si el profesional participa o no en esos ámbitos de decisión, esto es, según su autoridad (Sweep; Cameron; Weaver-Lariscy, 1994; Dozier; Broom, 2012, pp. 149-159).

Los roles son contingentes según el tipo de organización (sector, industria), la visión que tenga la dirección sobre la función de la comunicación organizacional, o según se trate de comunicación interna o externa (Moss; Warnaby; Newman, 2000; Moss; Green, 2002; Vieira; Grantham, 2015). 
Moss et al., con un estudio de alcance mundial, concluyen que al definir los roles profesionales se debe considerar que los factores organizacionales muchas veces responden a factores del entorno como el sistema cultural, económico y sociopolítico propio de cada país (Moss et al., 2017, p. 87).

Estos factores del entorno son muy significativos cuando se estudian los roles profesionales en América Latina. Por ejemplo, en Colombia el entorno de inestabilidad política orienta a los profesionales a un mayor desarrollo de sus funciones internas, y los altos índices de corrupción y falta de transparencia debilitan el trabajo que los profesionales se proponen en las relaciones entre organización y gobierno (Molleda; Suárez, 2003; 2005). En Chile, la influencia del régimen de Pinochet y la dinámica de la economía neoliberal marcó los valores propios de la cultura de las organizaciones y, por lo tanto, también el de los profesionales de la Comunicación Organizacional (Ferrari, 2003).

En una investigación publicada en 1982, Broom señaló diferencias significativas en el desempeño de los distintos roles según el sexo del profesional, ya que las mujeres se desempeñaban con más frecuencia que los varones en los roles técnicos y, según el autor, esta era causa de la diferencia de salarios entre ambos sexos (Broom, 1982, pp. 19-22). La incidencia del género en el desempeño de los roles profesionales orienta una parte muy importante de la investigación empírica desde la década de los años noventa (Toth, 1988; Toth et al., 1998).

\subsection{Rol del consultor}

El primer estudio sobre el rol de los consultores en comunicación fue el de Broom y Smith en 1979. Entre los factores que definieron para evaluar la relación entre consultores y clientes estaban (Broom; Smith, 1979, p. 53):

- eficacia del procedimiento aplicado por el consultor para resolver el problema del cliente;

- adecuación del producto final a las expectativas del cliente;

- cumplimiento de la tarea realizada;

- experiencia del consultor;

- disposición actitudinal del consultor;

- apropiación del producto o solución por parte del cliente para no generar dependencia con el consultor y la responsabilidad del consultor durante su servicio.

En el estudio sobre la percepción de la relación entre clientes y consultores realizado por Kakabadse, Louchart y Kakabadse (2006) exclusivamente desde la perspectiva de los consultores, los autores no observan diferencias en los roles entre quienes se definen como consultores, asesores o consejeros, pero sí hay diferencias marcadas entre estos y los que se definen como proveedores de servicios. Los primeros suelen trabajar con un nivel alto de incertidumbre, tanto del contexto como de la misma organización. Por ello, suelen realizar tareas de investigación y de análisis para satisfacer la demanda del cliente. En cambio, el proveedor de servicios responde a un diagnóstico (acertado o no) realizado por el cliente y a una formulación de la demanda relativamente precisa.

Preciado-Hoyos, Nivia-Flórez y Correales-Rivas (2017) encuentran en un estudio de gran alcance en Colombia que los consultores o profesionales de agencias realizan fundamentalmente tareas técnicas y de producción, pero no de definición de estrategias. Concluyen que en Colombia el principal factor de dificultad en la relación entre consultores y clientes es la falta de conocimiento que los primeros tienen sobre la organización del cliente y su sector.

\section{Funciones de la Comunicación Organizacional}

Con base en las teorías de la comunicación, es posible identificar tres dimensiones en las que opera la Comunicación Organizacional, y de las que derivan las funciones que persiguen los profesionales en el desempeño del rol.

- Dimensión de la información: se refiere a la generación, distribución, control de los datos que circulan dentro de la organización y entre esta y su entorno, tanto en forma dirigida a un público particular o abierta. También tiene la función de diseño y gestión de los procesos y canales para que las personas tengan la información necesaria en cantidad y calidad (transparencia, confiabilidad, claridad, oportunidad) para hacer su trabajo, para entenderlo y coordinarlo con otros y para que la organización opere y logre sus objetivos (Restrepo, 1995, p. 95; Hutton, 1999, p. 207; Van Ruler, 2004, p. 139).

- Dimensión de las relaciones: que Van-Ruler llama de "diálogo", se basa en generar relaciones y facilitarlas. Permite la socialización y la formación, y requiere identificar mutuos intereses, generar compromiso y cooperación tanto en públicos internos como externos (Restrepo, 1995, p. 95; Hutton, 1999, pp. 205-210; Van-Ruler, 2004, p. 139-140). Algunos autores añaden la función de construir consenso, que implica la escucha, el reconocimiento, darle la palabra al otro y asegurar la participación, y que por su naturaleza se puede incluir en esta dimensión ya que la completa con la comunicación de doble vía (Restrepo, 1995, p. 95; Van-Ruler, 2004, p. 139).

- Dimensión de la persuasión: que Restrepo define como “divulgación”, en el sentido de dar a conocer, hacer público, generar interés, persuadir a los públicos para que compartan una representación de lo que se ofrece. Esta dimensión está directamente vinculada con la influencia en los valores, actitudes y opiniones de las personas y, por tanto, en el clima organizacional, la motivación de las personas, la imagen y la reputación de la organización. Además, está fuertemente relacionada con la representación de la organización, desde su identidad visual hasta la defensa pública de sus líderes (Restrepo, 1995, p. 95; Hutton, 1999, pp. 207-208; Van-Ruler, 2004, p. 139). 
En el documento conocido como Melbourne Mandate -presentado en ocasión del World Public Relations Forum de 2012 a partir de una investigación de alcance mundial- se identifican áreas críticas para la función de los comunicadores organizacionales que forman parte de la dimensión persuasión y de las relaciones. Estas son (Gregory, 2015, p. 601):

- definir y mantener la identidad y los valores de la organización;

- construir una cultura de escucha y compromiso;

- instalar la responsabilidad social, organizacional, personal y profesional.

Molleda y Suárez (2003) y Molleda y Fergurson (2004) proponen una visión política de las Relaciones Públicas que focaliza la función social y de transformación del rol profesional. Esta visión es identificada como Escuela Latinoamericana de las Relaciones Públicas o, como también la denominan los autores, modelo colaborativo. Las funciones del rol del profesional están orientadas por la ética y la responsabilidad social, el bienestar de los empleados y de la comunidad, y la armonía con el gobierno. Hutton (1999, p. 207) se refiere a estos contenidos como "la persuasión relativa a causas", en la que prevalece el interés colectivo frente al individual u organizacional.

En ocasiones se discute si la comunicación interna es una función de la Comunicación Organizacional o es un campo independiente y, por lo general, la reflexión y la intervención sobre ella suele ser postergada frente a las exigencias de la comunicación externa (Almansa-Martínez, 2011, p. 95). En este estudio se entiende la comunicación interna como un ámbito propio de todas las funciones de la Comunicación Organizacional al igual que la comunicación externa.

En general los profesionales cuyos roles aplican a la comunicación interna se ocupan, entre otras tareas, de la gestión de la información, de las relaciones de la identidad y la cultura organizacional (Verčič; Verčič; Sriramesch, 2012, pp. 226227; Almansa-Martínez, 2011, pp. 97-100).

\section{La profesión en Uruguay}

En Uruguay, la institucionalización de la Comunicación Organizacional tiene varios hitos. En otros lugares del mundo la profesión tuvo su inicio en la primera mitad del siglo XX impulsada por las políticas gubernamentales, la propaganda, los conflictos sociales o por la influencia de las grandes empresas (Castillo-Esparcia, 2009, pp. 54-72). En cambio, en Uruguay los primeros registros de la profesión son de la década de los 60s y están directamente vinculados a las organizaciones públicas y, en particular, con tareas casi exclusivamente de ceremonial, protocolo y relación con la prensa. Con esta perspectiva se creó oficialmente en 1968 la Asociación Uruguaya de Relaciones Públicas.

En 1994 se inició el primer programa universitario en Comunicación Organizacional y con sus graduados comenzó el crecimiento dinámico y sostenido del ejercicio profesional en organizaciones privadas con y sin fines de lucro.

En 2004 surgió el primer posgrado en Comunicación Organizacional. Actualmente cuatro universidades (de un total de siete universidades oficiales) ofrecen formación de grado o postgrado en este campo.

Desde 2013 funciona además la Asociación Uruguaya de Comunicadores Organizacionales, cuya misión es la consolidación de la profesión en el país.

A pesar de que en Uruguay existe una asociación profesional con 50 años de historia y de que la primera formación universitaria cumplió 25 años, aún no se ha realizado ninguna investigación con base empírica que dé cuenta de la profesión en el país.

Este estudio tuvo como finalidad describir cómo es el desempeño del rol entre los profesionales de la Comunicación Organizacional en Uruguay y realizar por primera vez en América Latina una investigación que incluye todas las tareas y funciones de los comunicadores organizacionales diferenciadas según variables básicas y organizacionales.

\section{Metodología}

Esta investigación tuvo una fase exploratoria en la que se realizaron 9 entrevistas semiestructuradas, de aproximadamente 50 minutos cada una, a profesionales de la Comunicación Organizacional con más de 10 años de experiencia. Los entrevistados fueron seleccionados porque tenían experiencia variada en sus carreras profesionales en organizaciones públicas, privadas con y sin fines de lucro, y como asesores externos tanto en comunicación interna como externa. Es decir, son conocedores de los diferentes ámbitos de ejercicio de la profesión.

Las entrevistas permitieron ajustar las denominaciones de roles, tareas y funciones más habituales en las investigaciones revisadas, y con ello redactar de forma precisa las preguntas de la encuesta y dar contexto a las respuestas de los encuestados.

Con el resultado de las entrevistas se llegó a una definición de los términos para nombrar los roles profesionales que integra los tres principales modelos, incluye los roles de quienes trabajan como proveedores de servicios (consultores, agencias), relaciona los roles con las tareas que predominan en cada uno y completa los modelos definiendo el alcance de esos roles (tabla 3). 
Tabla 3. Roles y tareas del profesional de la Comunicación Organizacional

\begin{tabular}{|c|c|c|c|c|c|}
\hline Roles & Tareas & \multicolumn{2}{|c|}{ Dominios de las tareas } & \multicolumn{2}{|c|}{ Alcance de las tareas } \\
\hline Asesor & $\begin{array}{l}\text { Investiga, analiza para } \\
\text { diagnosticar y evaluar. } \\
\text { Capacita. }\end{array}$ & \multirow{3}{*}{$\begin{array}{l}\text { Se ocupa de asuntos } \\
\text { clave para la organiza- } \\
\text { ción, problemas, cri- } \\
\text { sis, conflictos, causas } \\
\text { sociales, soluciones, } \\
\text { oportunidades, públi- } \\
\text { cos, valores, fines. }\end{array}$} & \multirow{3}{*}{$\begin{array}{l}\text { Se ocupa de polí- } \\
\text { ticas, estrategias, } \\
\text { programas, planes, } \\
\text { proyectos, acciones, } \\
\text { recursos económicos, } \\
\text { tecnológicos y huma- } \\
\text { nos, presupuestos, } \\
\text { campañas. }\end{array}$} & Variable & $\begin{array}{l}\text { La responsabilidad está } \\
\text { delegada en los decisores. }\end{array}$ \\
\hline $\begin{array}{l}\text { Gerencial/ } \\
\text { decisor }\end{array}$ & $\begin{array}{l}\text { Analiza, negocia, planifi- } \\
\text { ca, organiza. }\end{array}$ & & & Largo plazo & $\begin{array}{l}\text { Tiene responsabilidad } \\
\text { final sobre decisiones y } \\
\text { resultados. }\end{array}$ \\
\hline $\begin{array}{l}\text { Gerencial/ } \\
\text { ejecutivo }\end{array}$ & $\begin{array}{l}\text { Organiza, administra, } \\
\text { supervisa, monitorea. }\end{array}$ & & & Medio plazo & $\begin{array}{l}\text { Tiene responsabilidad so- } \\
\text { bre los procesos y acciones. }\end{array}$ \\
\hline $\begin{array}{l}\text { Técnico } \\
\text { (operacional) }\end{array}$ & $\begin{array}{l}\text { Monitorea, implementa, } \\
\text { fabrica, produce, opera. }\end{array}$ & \multicolumn{2}{|c|}{$\begin{array}{l}\text { Genera contenidos, mediciones, reportes, } \\
\text { gráfica, actividades. }\end{array}$} & Corto plazo & $\begin{array}{l}\text { Tiene responsabilidad } \\
\text { acotada a los productos. }\end{array}$ \\
\hline Creador & Innova, aplica. & \multicolumn{2}{|c|}{ Genera nuevas ideas, medios, herramientas. } & Variable & $\begin{array}{l}\text { La responsabilidad es dele- } \\
\text { gada en los decisores. }\end{array}$ \\
\hline
\end{tabular}

Entre febrero y marzo de 2019 se realizó una encuesta online en la plataforma Qualtrics. En Uruguay no existe registro único de profesionales en este campo, por lo que el universo para la encuesta fue construido a base de estimaciones de las asociaciones profesionales y los graduados universitarios. Con esta información se elaboró una base inicial de contactos de 350 comunicadores organizacionales que se amplió con la técnica de bola de nieve. Los casos válidos -quienes efectivamente realizan tareas de Comunicación Organizacional en el momento de la encuesta- fueron 215.

La muestra tuvo las siguientes características:

- el $78 \%$ de los encuestados fueron mujeres y el $41 \%$ del total tenía entre 31 y 40 años;

- la casi totalidad (98\%) contaba con estudios universitarios de grado y $60 \%$ además con posgrado;

- el $60 \%$ tenía 10 o más años de experiencia en el campo de la comunicación organizacional;

- el 55\% tenía personal a cargo.

En el momento de realizar la encuesta el $80 \%$ ocupaba cargos dentro de organizaciones, $32 \%$ de ellos en organizaciones del sector público, $29 \%$ en organizaciones privadas con fines de lucro (empresas) y $19 \%$ en organizaciones privadas sin fines de lucro (tercer sector). El $20 \%$ de los encuestados restantes trabajaba como proveedor externo de servicios de Comunicación Organizacional, algunos como profesionales independientes (8\%) y otros en agencias de comunicación (12\%).

El 19\% de los encuestados pertenecía a organizaciones muy grandes (más de 1.000 empleados); el 25\% a grandes (entre 200 y 999 empleados); el 30\% a organizaciones medianas (entre 20 y 199 empleados); el 19\% a pequeñas (entre 5 y 19 empleados) y el $7 \%$ a micro organizaciones (hasta 4 empleados).

Para el $20 \%$ de los encuestados el principal responsable de la comunicación de su organización se encontraba en el nivel jerárquico más alto; para el $39 \%$ en el nivel jerárquico siguiente y para el $32 \%$ de los encuestados se encontraba en un nivel jerárquico intermedio de su organización.

El 18\% de los encuestados definió su unidad de Comunicación Organizacional como grande (11 o más empleados dentro de la unidad), en otro $18 \%$ de los casos la unidad de comunicación de su organización tenía entre 6 y 10 empleados, para el $23 \%$ entre 4 o 5 empleados, para el $26 \%$ entre 2 o 3 empleados y para el $16 \%$ de los encuestados las unidades de comunicación en su organización eran unipersonales ${ }^{1}$.

\section{Resultados}

Los profesionales varones de la Comunicación Organizacional en Uruguay, más que las profesionales mujeres, deciden sobre políticas o planes de largo plazo, toman decisiones que implican recursos muy variados (económicos, humanos, tecnológicos) que pueden afectar varios sectores de la organización, y son los responsables de los resultados de la comunicación, sean positivos o negativos. Es decir, los varones desempeñan el rol decisor en Comunicación Organizacional en mayor proporción que las mujeres (tabla 4).

En cambio, la mayoría de las profesionales mujeres desempeña roles ejecutivos en Comunicación Organizacional: organizan la implementación de programas y acciones de comunicación, coordinan los recursos y equipos, supervisan el trabajo y son responsables de que las acciones se realicen.

Los roles de asesoría -como aconsejar, orientar las decisiones que toman otros, buscar y analizar información para tomar decisiones sobre comunicación, capacitar personas, promover o facilitar la comunicación organizacional- predominan entre los profesionales que trabajan en agencias de comunicación (en la tabla 4 señalado como tipo de organización "externa").
Que la organización sea del sector público o privado determina cuál es el rol principal de los comunicadores organizacionales 
Los roles creativos como generar ideas nuevas, innovar en el uso de herramientas o medios de comunicación, proponer acciones de comunicación originales o novedosas predominan entre quienes trabajan como profesionales independientes (incluidos en tipo de organización "externa").

Tabla 4. Rol desempeñado según sexo y tipo de organización (\%)

\begin{tabular}{|c|c|c|c|c|c|c|}
\hline \multirow{2}{*}{ Rol } & \multicolumn{2}{|c|}{ Sexo } & \multicolumn{3}{|c|}{ Tipo de organización } & \multirow{2}{*}{ Todos } \\
\hline & Varones & Mujeres & Pública & Privada & Externa & \\
\hline Decisor & 42,6 & 21,5 & 20,3 & 26,0 & 37,5 & 26,0 \\
\hline Ejecutivo & 19,1 & 39,3 & 40,6 & 37,5 & 17,5 & 34,9 \\
\hline Técnico & 14,9 & 20,8 & 23,2 & 18,3 & 15,0 & 19,5 \\
\hline Asesor & 14,9 & 10,1 & 11,6 & 8,6 & 17,5 & 11,2 \\
\hline Creador & 8,5 & 8,3 & 4,3 & 9,6 & 12,5 & 8,4 \\
\hline Total & 100 & 100 & 100 & 100 & 100 & 100 \\
\hline
\end{tabular}

Por otra parte, los roles técnicos entre los profesionales de la Comunicación Organizacional en Uruguay son más frecuentes entre quienes ocupan cargos de menor jerarquía y sin personal a cargo. Algo similar se observa en los roles creativos que también predominan entre los profesionales con menor experiencia (tabla 5).

Por el contrario, el rol decisor, predomina entre los profesionales con mayor experiencia, en puestos de nivel jerárquico alto y con personal a cargo. El rol ejecutivo es claramente un rol de nivel jerárquico medio.

No se observan diferencias significativas entre roles según el tamaño de la unidad de comunicación a la que pertenece el encuestado, es decir, todos los roles están presentes tanto en las unidades pequeñas como en las grandes.

Tabla 5. Rol desempeñado según años de experiencia, nivel jerárquico y tenencia de personal a cargo (\%)

\begin{tabular}{|c|c|c|c|c|c|c|c|c|c|}
\hline \multirow{2}{*}{ Rol } & \multicolumn{3}{|c|}{ Años de experiencia } & \multicolumn{3}{|c|}{ Nivel jerárquico } & \multicolumn{2}{|c|}{ Personal a cargo } & \multirow{2}{*}{ Todos } \\
\hline & Menos de 5 & 6 a 9 & 10 o más & Bajo & Medio & Alto & Sin & Con & \\
\hline Decisor & 9,7 & 19,6 & 32,8 & 4,2 & 16,4 & 43,1 & 8,5 & 37,2 & 26,0 \\
\hline Ejecutivo & 29,0 & 36,0 & 35,9 & 27,1 & 54,6 & 35,4 & 35,4 & 43,0 & 34,9 \\
\hline Técnico & 29,0 & 26,8 & 14,1 & 45,8 & 12,7 & 7,7 & 32,9 & 8,1 & 19,5 \\
\hline Asesor & 9,7 & 10,7 & 11,7 & 4,2 & 14,5 & 9,2 & 11,0 & 8,1 & 11,2 \\
\hline Creador & 22,6 & 7,1 & 5,5 & 18,7 & 1,8 & 4,6 & 12,2 & 3,5 & 8,4 \\
\hline Total & 100 & 100 & 100 & 100 & 100 & 100 & 100 & 100 & 100 \\
\hline
\end{tabular}

El rol de decisor es claramente el deseado por la mayoría de los encuestados. De poder elegir, el $51 \%$ de los encuestados quisiera desempeñar ese rol en un futuro. Sin embargo, un 17\% de quienes actualmente tienen un rol de decisión desearían desempeñar roles creativos.

El tiempo dedicado a las diferentes tareas (tabla 6) se distribuye de manera consistente con el tipo de rol desempeñado. Quienes desempeñan roles de decisión o ejecutivos, están ubicados en los niveles jerárquicos altos de su organización, ocupan gran parte de su tiempo en coordinar equipos, liderar personas, planificar y presupuestar, evaluar estrategias y acciones de comunicación. Estas tareas ocupan además gran parte del tiempo de todos los profesionales encuestados que trabajan en organizaciones privadas.

En cambio, quienes desempeñan roles técnicos, están ubicados en los niveles jerárquicos bajos de su organización, dedican gran parte de su tiempo en diseñar nuevos medios o productos gráficos, explorar nuevas ideas, producir fotografías o audiovisuales.

Las tareas de organizar campañas, actividades y eventos, en las que hay un predominio de mujeres, son compartidas tanto por los roles ejecutivos como los técnicos y en particular es a la que le dedican más tiempo los profesionales de las organizaciones públicas incluidos los organismos internacionales.

Los profesionales de estas últimas organizaciones destinan más tiempo que sus colegas de organizaciones privadas a la gestión de prensa, el contacto con colegas y periodistas, la elaboración de comunicados y contenidos para redes y medios y su monitoreo.

Todos los profesionales dedican muy poco tiempo a analizar informes e investigaciones, definir objetivos, monitorear asuntos públicos y expectativas de los públicos, discutir visiones y estrategias con los directivos, y a evaluar escenarios.

\section{$42,6 \%$ de los comunicadores organiza-} cionales varones deciden sobre políticas y planes a largo plazo y sólo el $21,5 \%$ de las mujeres tiene ese rol 
Finalmente, las tareas de asesorar, advertir sobre riesgos, preparar para los medios, capacitar en comunicación para mejorar las competencias comunicativas de las personas, son a las que todos los profesionales dedican menos tiempo excepto quienes trabajan como profesionales independientes o en agencias.

Tabla 6. Principales tareas realizadas en una semana típica de trabajo, según orden de importancia por el tiempo dedicado y según predominio en segmentos.

\begin{tabular}{|c|c|c|c|c|c|}
\hline \multirow{2}{*}{\multicolumn{2}{|c|}{$\begin{array}{l}\text { Tareas en orden de importancia según tiempo dedica- } \\
\text { do a ellas (todos los encuestados) }\end{array}$}} & \multicolumn{4}{|c|}{ Las tareas predominan en: } \\
\hline & & \multirow{2}{*}{$\begin{array}{l}\text { Sexo } \\
\begin{array}{l}\text { Similar en } \\
\text { ambos }\end{array}\end{array}$} & \multirow{2}{*}{$\begin{array}{l}\text { Nivel jerárquico } \\
\text { Altos y medio }\end{array}$} & \multirow{2}{*}{$\begin{array}{r}\text { Rol } \\
\text { Decisory } \\
\text { ejecutivo }\end{array}$} & \multirow{2}{*}{$\begin{array}{l}\text { Tipo de organización } \\
\text { Privadas con y sin } \\
\text { fines de lucro }\end{array}$} \\
\hline $\begin{array}{c}1 \\
\text { Muy alta } \\
\text { dedicación }\end{array}$ & $\begin{array}{l}\text { Coordinar recursos, liderar, planificar, presu- } \\
\text { puestar, evaluar. }\end{array}$ & & & & \\
\hline 2 & Organizar campañas, actividades, eventos. & Mujeres & Bajos y medios & $\begin{array}{l}\text { Técnico y } \\
\text { ejecutivo }\end{array}$ & $\begin{array}{l}\text { Públicas y organismos } \\
\text { internacionales }\end{array}$ \\
\hline 3 & Diseñar, producir, explorar nuevas ideas. & $\begin{array}{l}\text { Similar en } \\
\text { ambos }\end{array}$ & Bajos & $\begin{array}{l}\text { Técnico y } \\
\text { creador }\end{array}$ & Similar en todas \\
\hline 4 & $\begin{array}{l}\text { Gestionar la relación con la prensa, elaborar } \\
\text { contenidos, monitorear redes o medios. }\end{array}$ & $\begin{array}{l}\text { Similar en } \\
\text { ambos }\end{array}$ & Similar en todos & $\begin{array}{l}\text { Similar en } \\
\text { todos }\end{array}$ & $\begin{array}{l}\text { Públicas y organismos } \\
\text { internacionales }\end{array}$ \\
\hline 5 & $\begin{array}{l}\text { Analizar informes, definir objetivos, discutir } \\
\text { estrategias. }\end{array}$ & $\begin{array}{l}\text { Similar en } \\
\text { ambos }\end{array}$ & Similar en todos & $\begin{array}{l}\text { Similar en } \\
\text { todos excepto } \\
\text { técnico }\end{array}$ & Similar en todas \\
\hline $\begin{array}{c}6 \\
\text { Muy baja } \\
\text { dedicación }\end{array}$ & Asesorar, advertir sobre riesgos, capacitar. & $\begin{array}{l}\text { Similar en } \\
\text { ambos }\end{array}$ & $\begin{array}{l}\text { Similar en todos, } \\
\text { algo más en niveles } \\
\text { altos y medios }\end{array}$ & Asesor & $\begin{array}{l}\text { Agencias y profesiona- } \\
\text { les independientes }\end{array}$ \\
\hline
\end{tabular}

El 79\% de los encuestados dice que en su organización se ha contratado algún servicio externo en los últimos dos años. La mayoría de los servicios contratados han sido de producción para la comunicación: diseño gráfico, fotografía, audiovisual, gestión o monitorización de prensa o de redes, organización de un evento o realización de productos publicitarios. Estos servicios se contratan principalmente en las organizaciones pequeñas o medianas. En menor medida las organizaciones contrataron servicios de asesoramiento en comunicación, ya sea estratégico o para la planificación. Y, por último, en algunas organizaciones se contrataron servicios de investigación de marca, de imagen, de clima interno, entre otros.

Los encuestados que han contratado servicios externos (identificados como "clientes" en la tabla 7) afirman que el principal factor que consideran para elegir un proveedor es la calidad de sus propuestas (tabla 7). Este factor tiene mucho peso para los encuestados de organizaciones privadas, con o sin fines de lucro (54\%), y para quienes contrataron servicios de investigación (63\%).

El segundo factor en importancia cuando se elige un proveedor es el precio o la cotización y este factor tiene mayor peso en las organizaciones públicas y en los organismos internacionales (52\%).

Por el contrario, quienes son proveedores de servicios opinan que el principal factor que sus clientes consideran a la hora de elegir un proveedor son las recomendaciones personales.

Para los encuestados que han contratado servicios externos, el principal factor de evaluación de un proveedor en Comunicación Organizacional (tabla 8) es su cumplimiento y responsabilidad, esto es especialmente importante para quienes trabajan en organizaciones públicas. Otros factores que también inciden, pero bastante más lejos que el primero, son la creatividad, la visión estratégica, el conocimiento que tenga el proveedor sobre el sector de la organización y el compromiso, empatía y adaptabilidad que logre con el cliente. La actualización y dominio tecnológico que tenga el proveedor prácticamente no son considerados por los encuestados: sólo 8 encuestados lo mencionan como un factor relevante para evaluar a un proveedor externo.

Por el contrario, los profesionales que prestan servicios a las organizaciones opinan que sus clientes priorizan la visión estratégica, el conocimiento que tengan sobre la organización o el entorno, pero no destacan como un factor relevante el cumplimiento y la responsabilidad.
Tabla 7. Principal factor en la elección de un proveedor externo según la opinión de clientes y proveedores (\%)

\begin{tabular}{|l|c|c|}
\hline \multicolumn{1}{|c|}{ Factor } & Clientes & Proveedores \\
\hline Precio, cotización & 37,0 & 30,0 \\
\hline Recomendaciones personales & 13,8 & 45,0 \\
\hline Imagen o marca del proveedor & 1,4 & 0,0 \\
\hline Propuesta que presenta & 47,8 & 25,0 \\
\hline Total & 100 & 100 \\
\hline
\end{tabular}

\section{Clientes y consultores en Comunicación} Organizacional difieren en qué es importante y qué problemático en la relación entre ambos 
Tabla 8. Principal factor de evaluación de los proveedores externos según la opinión de clientes y proveedores

\begin{tabular}{|c|c|c|}
\hline & \\
\hline & Clientes & Proveedores \\
\hline Cumplimiento, responsabilidad & 1 & 4 \\
\hline Creatividad innovación & 2 & 3 \\
\hline Compromiso, empatía & 3 & 2 \\
\hline Visión estratégica y conocimiento de la organización y entorno & 4 & 1 \\
\hline Aprendizaje y transferencia & 5 & 6 \\
\hline Productividad & 6 & 5 \\
\hline Actualización y dominio tecnológico & 7 & 7 \\
\hline
\end{tabular}

Al analizar los problemas y tensiones que se generan entre clientes y proveedores (tabla 9), los primeros consideran que las principales fuentes de tensiones son el no cumplimiento con fechas o entregas y la falta de conocimiento sobre el sector o los procesos de la organización.

En cambio, los proveedores encuentran que las principales fuentes de tensión con sus clientes son las diferentes expectativas sobre resultados, roles y tareas, y la poca claridad en los objetivos o el alcance.

Tabla 9. Principal factor de tensión en la relación cliente-proveedor, según la opinión de clientes y proveedores

\begin{tabular}{|l|c|}
\hline No cumplimiento con fechas y entregas & 1 \\
\hline Falta conocimiento sobre la organización o el sector & 2 \\
\hline Desempeño técnicamente pobre, con errores & 3 \\
\hline Diferencias en las expectativas sobre resultados, tareas & 4 \\
\hline Asignación de personal junior & 5 \\
\hline Poca claridad en objetivos y alcance & 6 \\
\hline Incompatibilidad de valores, normas & 2 \\
\hline Interpretaciones diferentes sobre situaciones o acciones & 8 \\
\hline Desacuerdos financieros & 8 \\
\hline Mal relacionamiento, diferencias personales & 6 \\
\hline
\end{tabular}

Según la opinión de la mayoría de los encuestados, en las organizaciones donde trabajan se priorizan las funciones de comunicación externa (tabla 10). En las organizaciones públicas se prioriza informar a los públicos externos sobre servicios y productos de la organización, sobre resultados y logros de la organización, y priorizan mejorar el acceso a la información pública y la transparencia. Las organizaciones privadas, con o sin fines de lucro, priorizan fortalecer la imagen externa y la reputación de la organización, realizar publicidad, propaganda o comunicación institucional algo más que las organizaciones públicas.

Sólo uno de cada cuatro encuestados dice que su organización prioriza la comunicación interna y en particular dentro de ella, la función de informar a los miembros de la organización sobre asuntos del trabajo y las tareas, sobre planes y resultados de la organización y facilitar la coordinación y circulación de información entre los miembros de la organización. La función interna tiene mayor prioridad en las organizaciones pequeñas y medianas.

Tabla 10. Función priorizada en la Comunicación Organizacional según tipo de organización (primera mención, \%)

\begin{tabular}{|c|c|c|c|}
\hline & Pública & Privada & Todas \\
\hline Informar a los públicos externos & 49,3 & 25,0 & 34,3 \\
\hline Fortalecer la imagen y reputación externa & 23,2 & 34,6 & 30,3 \\
\hline Fortalecer los vínculos y relaciones externas & 7,2 & 7,7 & 7,4 \\
\hline Cuidar la identidad visual y la marca, representar públicamente a la organización & 4,3 & 1,9 & 2,9 \\
\hline Informar a los miembros internos & 13,0 & 13,5 & 13,1 \\
\hline Fortalecer la identidad y pertenencia de los miembros, reforzar valores y actitudes de los miembros & 1,4 & 13,5 & 8,6 \\
\hline Fortalecer los vínculos y relaciones internas & 1,4 & 4,8 & 3,4 \\
\hline Total & 100 & 100 & 100 \\
\hline
\end{tabular}

En la tabla 11 se presentan los mismos resultados reagrupados según refieran al ámbito de comunicación interna o externa de las organizaciones y según la dimensión comunicacional a la que aportan: información, relaciones o persuasión. Se observa que la preocupación por las relaciones es significativamente más baja tanto en el ámbito interno como externo. En muy pocas organizaciones se prioriza el involucramiento con los actores clave de la sociedad, fortalecer las relaciones y la fidelidad de los públicos externos y la comunidad. Y menos aún se preocupan por los vínculos entre los miembros de la organización, de estimular la participación, el diálogo, mediar o facilitar el entendimiento con los miembros. 
Esto es consistente con la evaluación global que hacen los encuestados sobre los resultados de la comunicación interna y la externa de sus organizaciones (tabla 12). Cerca de la mitad de los encuestados entienden que la comunicación interna en la organización donde trabajan no es exitosa. La comunicación interna es peor evaluada en las organizaciones del sector público que en las del sector privado.
Tabla 11. Función priorizada en la Comunicación Organizacional según ámbito y dimensión de la comunicación (\%)

\begin{tabular}{|l|c|c|c|}
\hline \multicolumn{1}{|c|}{ Dimensión } & $\begin{array}{c}\text { Comunicación } \\
\text { interna }\end{array}$ & $\begin{array}{c}\text { Comunicación } \\
\text { externa }\end{array}$ & Total \\
\hline Información & 13,1 & 34,3 & 47,4 \\
\hline Persuasión & 8,6 & 33,1 & 41,8 \\
\hline Relaciones & 3,4 & 7,4 & 10,8 \\
\hline Total & 25,1 & 74,9 & 100 \\
\hline
\end{tabular}

Tabla 12. Evaluación de la Comunicación Organizacional interna y externa según tipo de organización (en porcentajes)

\begin{tabular}{|l|c|c|c|c|c|c|}
\cline { 2 - 7 } \multicolumn{1}{c|}{} & \multicolumn{3}{c|}{ Comunicación interna } & \multicolumn{3}{c|}{ Comunicación externa } \\
\cline { 2 - 8 } \multicolumn{1}{c|}{} & Pública & Privada & Todas & Pública & Privada & Todas \\
\hline Evaluación positiva & 20,4 & 33,7 & 32,0 & 50,0 & 67,3 & 62,9 \\
\hline Evaluación neutra & 20,4 & 28,7 & 26,0 & 22,2 & 25,7 & 24,6 \\
\hline Evaluación negativa & 59,2 & 37,6 & 41,9 & 27,8 & 5,0 & 12,6 \\
\hline Total & 100 & 100 & 100 & 100 & 100 & 100 \\
\hline
\end{tabular}

\section{Discusión y conclusiones}

Como surge de otras investigaciones empíricas sobre Comunicación Organizacional en el mundo, en Uruguay los profesionales tienen un rol principal, pero hacen "un poco de todo" y los roles que desempeñan varían de acuerdo con factores organizacionales y personales.

En los resultados de esta investigación se observa que el rol clásico gerencial muestra dos perfiles:

- decisor, en el que predomina la toma de decisiones sobre políticas y planes a largo plazo y la responsabilidad última del profesional es sobre los resultados de la comunicación;

- ejecutivo, cuya prioridad está en la organización de los recursos para lograr los objetivos y por tanto la responsabilidad del profesional está en la realización y logros de las acciones o actividades.

Ambos perfiles requieren competencias diferentes, el primero requiere pensamiento analítico y sistémico, entre otras competencias y el segundo, un gran dominio de las relaciones interpersonales y grupales.

El rol decisor es el que más desempeñan quienes ocupan niveles jerárquicos altos en sus organizaciones y es más frecuente entre varones. El rol ejecutivo predomina en los niveles jerárquicos intermedios y es más frecuente entre las mujeres, quienes consistentemente realizan más que los varones tareas relativas a la organización de campañas, actividades y eventos. Esto muestra una situación similar a la que observaba Toth et al. (1998) desde la década de los años 90 en Estados Unidos y Reino Unido. Un estudio posterior a este podría triangular la percepción de los profesionales, sus colegas y sus empleadores sobre esta disparidad entre sexo, para explorar si estos resultados se deben a factores personales de los propios profesionales o a factores culturales de la sociedad, la organización o los empleadores.

Casi todos los profesionales hacen algo de producción de contenidos o de monitorización de medios, pero el rol técnico es propio de los profesionales que ocupan cargos jerárquicos más bajos y no tienen personal a cargo. En este caso la responsabilidad del profesional está acotada a lo inmediato: la realización de un producto o la prestación directa de un servicio. Los profesionales que tienen principalmente el rol de creador tienen un perfil similar al del técnico, pero es desempeñado con más frecuencia por profesionales que trabajan en forma independiente. Es un perfil de rol que se debería estudiar con mayor profundidad, ya que se trata de profesionales que realizan tareas o tienen competencias requeridas para los procesos de innovación y el emprendedurismo y es además un rol que una parte de los profesionales de más alto nivel desearían desempeñar.

El rol de asesor es más frecuente entre los proveedores externos y entre los profesionales que ocupan niveles más altos en la organización. Este rol incluye entre sus principales tareas las de analizar, evaluar, discutir y asesorar. Como son tareas menos requeridas en el flujo regular de gestión de la comunicación son postergadas, ocupan un tiempo marginal de los profesionales que trabajan en cargos organizacionales y para cumplir con ellas contratan servicios externos.

Desempeñar uno u otro rol no depende del tamaño de la unidad de Comunicación Organizacional, pero sí del nivel jerárquico que ocupa el profesional, si tiene o no personal a cargo y los años de experiencia. Las tres variables se refieren a la carrera del profesional: con más experiencia existe mayor probabilidad de ocupar cargos de alto nivel y con estos, mayor probabilidad de tener personal a cargo. Si además se toma en cuenta que uno de cada dos profesionales que no tienen roles de decisor los desearían desempeñar, podríamos definir una dinámica de carác-

La comunicación interna tiene resultados negativos en la mayoría de las organizaciones y suele ser postergada frente a las exigencias de la comunicación externa 
ter típico que inicia con el rol técnico en la carrera, continúa con el ejecutivo y finaliza con el rol decisor. Para confirmar esta afirmación es necesario un estudio longitudinal de las carreras de los profesionales que permita identificar cómo intervienen estos y otros factores, tales como el tipo de organización o las competencias personales, en el paso de un rol a otro.

El tipo de organización, su misión y naturaleza, marca diferencias importantes en el tiempo que le dedican los profesionales a unas u otras tareas. En las organizaciones del sector público los presupuestos están definidos centralmente, existe bajo margen de negociación interna sobre los recursos y las normativas rigen de manera restrictiva el funcionamiento interno, la descripción de cargos y sus responsabilidades. En cambio, en las organizaciones privadas la negociación por el presupuesto es constante, la administración de los recursos tiene restricciones más exigentes y el liderazgo en personas y equipos es clave para lograr la dinámica y funcionamiento interno. Esto explica por qué en las organizaciones públicas los profesionales le dedican menos tiempo que sus colegas de las privadas a coordinar equipos, liderar personas, planificar y presupuestar.

En otro sentido, la relevancia que tiene la opinión pública en la reputación de las organizaciones públicas, la importancia particular que en las organizaciones públicas de Uruguay tienen la transparencia en la rendición de cuentas y la facilidad en el acceso a la información pública hacen que en las organizaciones de este sector predominen las tareas de organización de campañas, eventos y también la gestión de prensa, el contacto con periodistas, la elaboración de contenidos y el monitoreo de medios y redes.

Con respecto a la contratación de proveedores externos, las principales contrataciones se realizan para contenidos de roles técnicos: diseño gráfico, fotografía, audiovisual, gestión o monitorización de prensa o redes, organización de un evento o realización de productos publicitarios.

La diferente percepción que tienen clientes y proveedores sobre su relación es llamativa. Los primeros consideran el cumplimiento formal, en plazo, como el principal factor para evaluar un proveedor y el principal problema que encuentran en la relación con estos. Por el contrario, los profesionales que son proveedores de servicios no reconocen este aspecto como problemático.

También llama la atención que los clientes deciden la contratación de los proveedores por la propuesta que les presentan, pero desde la perspectiva de los proveedores, la propuesta es el tercer factor en importancia por la que son contratados. Si se toma en consideración que los proveedores opinan que las principales dificultades en la relación con sus clientes son la diferencia entre expectativas sobre resultados y la poca claridad en objetivos y alcance, se puede concluir que es necesario mejorar las competencias de consultores para elaborar las propuestas. Es allí donde se elabora con precisión la demanda, es la etapa en la que se ajustan expectativas de ambas partes, se eliminan ambigüedades y se puede bajar la incertidumbre en la relación entre las partes.

Con respecto a las funciones de la comunicación organizacional, la prioridad absoluta está en la comunicación externa. Vieira y Grantham (2015) en un estudio en Estados Unidos encuentran que los profesionales de las organizaciones públicas focalizan su atención en proveer información a los públicos más que los colegas de empresas privadas (p. 546). En esta investigación se observa algo similar. Las organizaciones públicas priorizan informar a sus públicos externos y mejorar el acceso a la información. En cambio, las organizaciones del sector privado priorizan fortalecer su imagen y reputación.

Como afirma Almansa-Martínez (2011, p. 95) la comunicación interna es la gran olvidada y, especialmente en Uruguay, esto sucede en las organizaciones grandes y del sector público. Además, es la función que recibe peor evaluación entre los profesionales uruguayos encuestados. Ruck y Welsch (2012) en una revisión de 12 estudios empíricos, afirman que la satisfacción con la comunicación interna se encuentra en niveles bajos de entre 50 y $60 \%$ de satisfacción (p. 301). Esto marca la relevancia de continuar estudiando la Comunicación Organizacional interna y en particular explorar si la causa de esta poca atención es por las dificultades propias de las tareas de comunicación interna, la falta de competencias específicas de los profesionales para trabajar en ella o por otra razón.

\section{Nota}

1. Moss et al. (2017) muestran en un estudio de alcance mundial que el tamaño de la unidad típica de Comunicación Organizacional es de 10 o menos empleados y que las decisiones funcionales clave (como la toma de decisiones, la asignación de responsabilidades y la organización de tareas en equipos) son esenciales y están presentes tanto en unidades pequeñas como en las muy grandes (pp. 88-89).

\section{Referencias}

Almansa-Martínez, Ana (2011). Del gabinete de prensa al gabinete de comunicación. La dirección de comunicación en la actualidad. Sevilla: Comunicación social ediciones y publicaciones. ISBN: 9788492860906

Arzuaga-Williams, Mónica (2017). "Revisión sobre el rol del profesional de las relaciones públicas". Austral comunicación, v. 6, n. 2, pp. 253-275.

https://doi.org/10.26422/AUCOM.2017.0602.ARZ 
Arzuaga-Williams, Mónica (2019). “Perspectivas latinoamericas sobre el rol de los profesionales de la comunicación organizacional y las relaciones públicas". Anagramas, v. 17, n. 34, pp 135-154.

https://doi.org/10.22395/angr.v17n34a7

Berkowitz, Dan; Hristodoulakis, Ilias (1999). "Practitioner roles, public relations education, and professional socialization: An exploratory study". Journal of public relations research, v. 11, n. 1, pp. 91-103.

https://doi.org/10.1207/s1532754xjprr1101_04

Broom, Glen M. (1982). "A comparision of sex roles in public relations". Public relations review, v. 8, n. 3, pp 17-22. https://doi.org/10.1016/S0363-8111(82)80028-3

Broom, Glen M.; Dozier, David M. (1986). “Advancement for public relations role models”. Public relations review, v. 12, n. 1, pp. 37-56.

https://doi.org/10.1016/S0363-8111(86)80039-X

Broom, Glen M.; Smith, George D. (1979). “Testing the practitioner's impact on clients”. Public relations review, v. 5, n. 3, pp. $47-59$. https://doi.org/10.1016/S0363-8111(79)80027-2

Castillo-Esparcia, Antonio (2009). Relaciones públicas. Teoría e historia. Barcelona: UOC. ISBN: 9788497888172

DeSanto, Barbara; Moss, Danny (2005). "Rediscovering what PR managers do: Rethinking the measurement of managerial behavior in the Public Relations context". Journal of communication management, v. 9, n. 2, pp. 179-196. https://doi.org/10.1108/13632540510621371

Dozier, David M.; Broom, Glen M. (1995). "Evolution of the manager role in Public Relations practice”. Journal of public relations research, v. 7, n. 1, pp. 3-26.

https://doi.org/10.1207/s1532754xjprr0701_02

Dozier, David M.; Broom, Glen M. (2012). "The centrality of practitioner roles to public relations theory". In: Botan, Carl H.; Hazleton, Vincent. Public relations theory II. New York: Routledge, pp. 137-170. ISBN: 0805833846

Ferrari, Maria-Aparecida (2003). "Public relations in Chile: Searching for identity amid imported models". En: Sriramesh, Krishnamurthy; Verčič, Dejan. The global public relations handbook. Theory, research, and practice. New Jersey: Lawrence Erlbaum Associates, pp. 378-395. ISBN: 0805839232

Gregory, Anne (2015). “Practitioner-leaders' representation of roles: The Melbourne Mandate”. Public relations review, v. 41 , n. 5, pp. 598-606.

https://doi.org/10.1016/j.pubrev.2014.02.030

Hutton, James G. (1999). "The definition, dimensions and domain of public relations". Public relations review, v. $25, \mathrm{n}$. 2, pp. 199-214.

https://doi.org/10.1016/S0363-8111(99)80162-3

Kakabadse, Nada K.; Louchart, Eddy; Kakabadse, Andrew (2006). "Consultant's role: a qualitative inquiry from the consultant's perspective". Journal of management development, v. 25, n. 5, pp. 416-500.

https://doi.org/10.1108/02621710610666268

Krohling-Kunsch, Margarida-Maria (2004). “A função das Relações Públicas e a prática comunicacional nas organizações". Organicom, v. 1, n. 1, pp. 121-139.

https://doi.org/10.11606/issn.2238-2593.organicom.2004.138874

Latin American Communication Monitor.

http://latincommunicationmonitor.com/site/informes

Mellado, Claudia; Barría, Sergio (2012). "Development of professional roles in the practice of public relations in Chile". Public relations review, v. 38, n. 3, pp. 446-453.

https://doi.org/10.1016/j.pubrev.2012.04.001

Molleda, Juan-Carlos; Fergurson, Mary-Ann (2004). "Public relations roles in Brazil: Hierarchy eclipses gender differences". Journal of public relations research, v. 16, n. 4, pp. 327-351.

https://doi.org/10.1207/s1532754xjprr1604_1

Molleda, Juan-Carlos; Moreno, Ángeles; Athaydes, Andréia; Suárez, Ana-María (2010). “Macroencuesta latinoamericana de comunicación y relaciones públicas". Organicom, v. 7, n. 13, pp. 118-141.

https://doi.org/10.11606/issn.2238-2593.organicom.2010.139074

Molleda, Juan-Carlos; Suárez, Ana-María (2003). "El papel de los profesionales colombianos de relaciones públicas como agentes de transformación social: cómo la crisis del país obliga a los profesionales a ir más allá de la comunicación con los públicos organizacionales". Anagramas, v. 2, n. 3, pp. 86-134.

https://revistas.udem.edu.co/index.php/anagramas/article/view/1244 
Molleda, Juan-Carlos; Suárez, Ana-María (2005). “Challenges in Colombia for Public Relations professionals: a qualitative assessment of the economic and political environments". Public relations review, v. 31, n. 1, pp. 21-29. https://doi.org/10.1016/j.pubrev.2004.10.001

Moss, Danny; Green, Rob (2002). "Re-examining the manager's role in public relations: What management and public relations research". Journal of communication management, v. 6, n. 2, pp. 118-132. https://doi.org/10.1108/13632540210806982

Moss, Danny; Likely, Fraser; Sriramesh, Krishnamurthy; Ferrari, Maria-Aparecida (2017). "Structure of the Public Relations/communication department: Key findings from a global study". Public relations review, v. 43, n. 1, pp. 80-90. https://doi.org/10.1016/j.pubrev.2016.10.019

Moss, Danny; Newman, Andrew; DeSanto, Barbara (2005). "What do communication managers do? Defining and refining the core elements of management in Public Relations/corporate communication context". Journalism \& mass communication quarterly, v. 82, n. 4, pp. 873-890.

https://doi.org/10.1177/107769900508200408

Moss, Danny; Warnaby, Gary; Newman, Andrew J. (2000). "Public relations practitioner role enactment at the senior management level within U.K. companies". Journal of public relations research, v. 12, n. 4, pp. 277-307. https://doi.org/10.1207/S1532754XJPRR1204_1

Preciado-Hoyos, Ángela; Nivia-Flórez, Adriana-Marcela; Correales-Rivas, Juan-Pablo (2017). "The strategic orientation of communications consulting firms in Colombia". Public relations review, v. 43, n. 2, pp. 406-416.

https://doi.org/10.1016/j.pubrev.2017.02.003

Restrepo, Mariluz (1995). “Comunicación para la dinámica organizacional”. Signo y pensamiento, v. 14, n. 26, pp. 91-96. https://revistas.javeriana.edu.co/index.php/signoypensamiento/article/view/3379

Ruck, Kevin; Welch, Mary (2012). "Valuing internal communication; management and employee perspectives". Public relations review, v. 38, n. 2, pp. 294-302.

https://doi.org/10.1016/j.pubrev.2011.12.016

Salas-Forero, Claudia-Patricia (2013). "Posicionamiento de la comunicación estratégica como gestión gerencial en las empresas más grandes de Colombia". Signo y pensamiento, v. 32, n. 63, pp. 94-109.

https://revistas.javeriana.edu.co/index.php/signoypensamiento/article/view/6944

Sweep, Duane; Cameron Glen T.; Waver-Lariscy, RuthAnn (1994). "Rethinking constraints on public relations practice". Public relations review, v. 20, n. 4, pp. 319-331.

https://doi.org/10.1016/0363-8111(94)90093-0

Toth, Elizabeth L. (1988). "Making peace with gender issues in Public Relations". Public relations review, v. 14, n. 3, pp. 36-47.

https://doi.org/10.1016/S0363-8111(88)80046-8

Toth, Elizabeth L.; Serini, Shirley A.; Wright, Donald K.; Emig, Arthur G. (1998). "Trends in public relations roles: $1990-$ 1995". Public relations review, v. 24, n. 2, pp. 145-163.

https://doi.org/10.1016/S0363-8111(99)80048-4

Van-Ruler, Betteke (2004). "The communication grid: An introduction of a model of four communication strategies". Public relations review, v. 30, n. 2, pp. 123-143.

https://doi.org/10.1016/j.pubrev.2004.01.002

Van-Ruler, Betteke; Verčič, Dejan; Bütschi, Gerhard; Flodin, Bertil (2004). "A first look for parameters of public relations in Europe". Journal of public relations research, v. 16, n. 1, pp. 35-63.

https://doi.org/10.1207/s1532754xjprr1601_2

Verčič, Ana-Tkalac; Verčič, Dejan; Sriramesh, Krishnamurthy (2012). “Internal communication: Definition, parameters, and the future". Public relations review, v. 38, n. 2, pp. 223-230.

https://doi.org/10.1016/j.pubrev.2011.12.019

Vieira, Edward T.; Grantham, Susan (2015). “Determining factors leading to strategic management PR practitioner roles". Public relations review, v. 41, n. 4, pp. 544-550.

https://doi.org/10.1016/j.pubrev.2015.05.005

Yeo, Su-Lin; Sriramesh, Krishnamurthy (2009). "Adding value to organizations: An examination of the role of senior public relations practitioners in Singapore". Public relations review, v. 35, n. 4, pp. 422-425.

https://doi.org/10.1016/j.pubrev.2009.08.003 